\title{
A SURVEY OF SELECTED CATEGORICAL PROPERTIES OF ALGEBRAS WITH MULTIVALUED ADDITION
}

\author{
WOJCIECH BORCZYK \\ Institute of Computer Science, University of Silesia \\ ORCID:0000-0001-5731-2345_E-mail:wojciech.borczyk@us.edu.pl \\ PAWEŁ G€ADKI \\ Institute of Mathematics, University of Silesia \\ ORCID: 0000-0001-5894-650X E-mail: pawel.gladki@us.edu.pl \\ KRZYSZTOF WORYTKIEWICZ \\ Laboratoire de Mathématiques, Université Savoie Mont Blanc \\ E-mail: krzysztof.worytkiewicz@univ-smb.fr
}

\begin{abstract}
In this paper we survey selected categorical properties of module-like objects with multivalued addition. The general theme of the results included here is that they are aimed towards designing a version of homological algebra over such structures. A vast majority of these results has been already known for some time, in a few instances where the authors could not find references, they provide short proofs in order to fill gaps in their exposition.
\end{abstract}

1. Introduction. Algebras with multivalued binary operations have been investigated by numerous researchers with respect to all sorts of applications. From the authors' point of view, of particular interest are the ones related in one way or another to axiomatic approaches to the algebraic theory of quadratic forms: real reduced hyperfields introduced by Marshall [19] provide an axiomatization of the (reduced) theory of quadratic forms in a first-order language. Unlike "classical" theory of quadratic forms, axiomatic theories are lacking the theory of cohomological invariants - it is thus tempting to investigate

2010 Mathematics Subject Classification: 20N20, 16Y99, 18A35.

Key words and phrases: hypergroups, hyperrings, hypermodules, categories of hypermodules. The paper is in final form and no version of it will be published elsewhere. 
if a version of homological algebra can be built, where hypermodules, hyperrings and hyperfields play roles similar to modules, rings and fields.

In this survey we review selected categorical properties of hypermodules that are needed for developing foundations of such homological algebra. Firstly, we start with listing all familiar definitions of algebras endowed with hyperoperations, and gradually reduce our considerations to a few categories of objects resembling modules with a few different notions of morphisms. We then proceed to discussing various characterizations of monics and epics, and prove variants of Homomorphiesatz wherever possible. In subsequent sections, we focus on products, coproducts, free objects, subobjects and quotient objects. Finally, we discuss equalizers and kernels, as well as coequalizers and cokernels, and conclude this survey with a result stating that the category of hypermodules with strong homomorphisms (possibly multivalued - see below for precise definitions) is Puppe exact, that is has zero objects, kernels and cokernels, is normal and conormal, and such that every morphism admits an epi-mono factorization. This is probably as close as one can get to the notion of Abelian categories for hypermodules.

The results in this paper accumulated over the past few years when the authors were trying to learn something about categories of hypermodules. A more careful investigation usually proved that whatever they were "discovering" had been already known and published in one form or another, most notably by very prolific Persian school of mathematicians and computer scientists working on hyperstructures. The reader will clearly see this when looking at the cited sources. A few examples and proofs that appear here are due to the authors, there is, however, no claim that these results are original: rather than that, the authors believe that they were simply not able to find proper references. This, as well as the fact that the results cited here are scattered over a plethora of papers, sometimes published in hard-to-find sources, convinced the authors that it is perhaps worthwhile to compile a survey like this, and the proceedings of the ALANT5 meeting in Będlewo is probably a good place where it can be presented to a broader audience.

The authors would like to express their thanks to the anonymous referee for a handful of valuable suggestions that made their presentation more comprehensible.

2. Basic definitions. Throughout the paper we shall denote by $\mathcal{P}^{*}(H)$ the set of all nonempty subsets of a set $H$.

Definition 2.1. Let $H \neq \emptyset$. A map $+: H \times H \rightarrow \mathcal{P}^{*}(H)$ is called a hyperoperation. A hyperoperation $+: H \times H \rightarrow \mathcal{P}^{*}(H)$ is called weak associative (see [34], 35]) if

$$
\forall a, b, c \in H[(a+b)+c \cap a+(b+c) \neq \emptyset]
$$

associative if

$$
\forall a, b, c \in H[(a+b)+c=a+(b+c)]
$$

weak commutative if

$$
\forall a, b \in H[a+b \cap b+a \neq \emptyset]
$$

commutative if

$$
\forall a, b \in H[a+b=b+a],
$$


and is said to satisfy the reproduction axiom if

$$
\forall a \in H[a+H=H=H+a],
$$

where $a+H=\bigcup_{h \in H} a+h,{ }^{1}$ and to be simplifiable on the left (right) if

$$
\forall a, b, c \in H[a+b \cap a+c \neq \emptyset \Rightarrow b=c] \quad(\forall a, b, c \in H[b+a \cap c+a \neq \emptyset \Rightarrow b=c]) .
$$

An element $0_{L} \in H\left(0_{R} \in H\right.$, respectively) is called a left scalar identity (a right scalar identity, respectively) if

$$
\forall a \in H\left[0_{L}+a=\{a\}\right] \quad\left(\forall a \in H\left[a+0_{R}=\{a\}\right], \text { respectively }\right),
$$

and an element $0 \in H$ is called a scalar identity if it is both a left and a right scalar identity. If $0 \in H$ is a scalar identity and $a \in H$, an element $-a \in H$ is called an inverse of $a$ if

$$
0 \in a+(-a) \cap(-a)+a .
$$

Finally, if $0 \in H$ is a scalar identity and for every element $a \in H$ there is an element $-a \in H,+$ is said to satisfy the exchange axiom if

$$
\forall a, b, c \in H[a \in b+c \Rightarrow b \in a+(-c)] .
$$

Definition 2.2. Let $H \neq \emptyset$ and let $+: H \times H \rightarrow \mathcal{P}^{*}(H)$ be a hyperoperation. The algebra $(H,+)$ is called:

(1) an $H_{v}$-semigroup, if + is weak associative.

(2) a semihypergroup if + is associative.

(3) a quasihypergroup if + satisfies the reproduction axiom.

(4) a hypermonoid if it is a semihypergroup with a scalar identity.

(5) an $(C O W) H_{v}$-group if it is an (weak commutative) $H_{v}$-semigroup and a quasihypergroup.

(6) a (commutative) hypergroup if it is a (commutative) semihypergroup and a quasihypergroup.

(7) a regular hypergroup if it is a hypergroup with a scalar identity such that for every element $a \in H$ there is a (not necessarily unique) inverse element $-a \in H$.

(8) a reversible regular hypergroup if it is a regular hypergroup and the exchange axiom is satisfied.

(9) a polygroup if it is a hypermonoid such that for every element $a \in H$ there is a unique inverse element $-a \in H$ and the exchange axiom is satisfied.

(10) a canonical hypergroup if it is a commutative polygroup.

For a semihypergroup $(H,+)$ a subset $\emptyset \neq A \subseteq H$ is called a complete part of $H$ if

$$
\forall n \in \mathbb{N} \forall a_{1}, \ldots, a_{n} \in H\left[\sum_{i=1}^{n} a_{i}+A \neq \emptyset \Rightarrow \sum_{i=1}^{n} a_{i} \subseteq A\right],
$$

\footnotetext{
${ }^{1}$ In general, for a hyperoperation + on the set $H$ and $A, B \subseteq H$, we shall write $A+B$ to denote the set

$$
A+B=\bigcup_{a \in A, b \in B} a+b
$$
}


the intersection of all the complete parts of $H$ is called a complete closure of $A$ and denoted by $\mathcal{C}(A)$, and $(H,+)$ is called a complete semihypergroup if

$$
\forall a, b \in H[\mathcal{C}(a+b)=a+b] .
$$

REMARK 2.3. Hypergroups were first considered by Marty [20] during the 8th Congress of Scandinavian Mathematicians when he defined a hypergroup as a set equipped with an associative and reproductive hyperoperation. Among other things, he has shown that a hypergroup simplifiable on the left (or on the right) is a group [20. Later a simpler proof of the same fact was given by Koskas [15], and Leoreanu [17] proved that a semihypergroup simplifiable on the left (or on the right) that satisfies the following weaker version of the reproduction axiom:

$$
\forall a \in H[a+H=H] \text { and } \exists a_{0} \in H\left[H=H+a_{0}\right]
$$

is a group. Note that, unlike in the group theory, hypergroups need not be hypermonoids, i.e. here associativity and reproductivity are not enough to infer the existence of a scalar identity.

Canonical hypergroups appeared for the first time in 1956 in the works by Krasner [16] on approximations of valued fields. Regular, reversible regular and complete hypergroups were introduced by Corsini [5] and Koskas [15] in the early 1970s. Every complete hypergroup is a regular reversible hypergroup [8, Theorem 46] and there are examples of regular hypergroups that are not reversible [7. Polygroups were introduced by Corsini [6] (there called quasicanonical hypergroups) around 1980 and later independently by Comer 4. $H_{v^{-}}$-structures were first introduced by Vougiouklis in 1990 at the 4th Algebraic Hyperstructures and Applications Congress [38].

For a detailed account on the theory of hypergroups in various flavors see the monographs [8], 9] and [10].

Definition 2.4. Let $H \neq \emptyset$ and let $+, \cdot: H \times H \rightarrow \mathcal{P}^{*}(H)$ be two hyperoperations.

(1) The algebra $(H,+, \cdot)$ is called a general $H_{v}$-ring if $(H,+)$ is an $H_{v}$-group, $(H, \cdot)$ is an $H_{v}$-semigroup and $\cdot$ is weak distributive with respect to + , i.e.

$$
\forall a, b, c \in H[a(b+c) \cap(a b+a c) \neq \emptyset \wedge(b+c) a \cap(b a+c a) \neq \emptyset] .
$$

A general $H_{v}$-ring might be weak commutative with respect to either + or $\cdot$, or both of them. We shall often abbreviate and write " $H_{v}$-ring" instead of "general $H_{v}$-ring". If only + is multivalued, then $H$ is called an additive $H_{v}$-ring. If only · is multivalued, then $H$ is called a multiplicative $H_{v}$-ring.

(2) The algebra $(H,+, \cdot)$ is called a general hyperring if $(H,+)$ is a hypergroup, $(H, \cdot)$ is a semihypergroup and $\cdot$ is distributive with respect to + , i.e.

$$
\forall a, b, c \in H[a(b+c)=a b+a c \wedge(b+c) a=b a+c a] .
$$

A general hyperring might be commutative with respect to either + or $\cdot$, or both of them. We shall often abbreviate and write "hyperring" instead of "general hyperring". If only + is multivalued, then $H$ is called an additive hyperring. If only · is multivalued, then $H$ is called a multiplicative hyperring. 
(3) The algebra $(H,+, \cdot)$ is called a Krasner hyperring if it is an additive hyperring such that $(H,+)$ is a canonical hypergroup with the scalar identity 0 such that

$$
\forall a \in H[a 0=0 a=0] .
$$

A Krasner hyperring is commutative if - is commutative.

REMARK 2.5. Krasner hyperrings were introduced by Krasner [16] in 1956 and were extensively studied by his students, most notably by Mittas [23] and Stratigopoulos [36]. The notion of general hyperrings was introduced in mid-1980s by De Salvo [12] and Vougiouklis [14. Multiplicative hyperrings were the object of studies by Procesi [29] and Rota [31] around the same time. $H_{v}$-rings were introduced by Vougiouklis [38 together with $H_{v}$-groups in 1990 . For more details on the general theory of hyperrings, Krasner hyperrings and $H_{v}$-rings see the monograph [11].

DEFINITION 2.6.

(1) Let $H$ be an $H_{v}$-ring. A COW $H_{v}$-group $(M,+)$ is called a left $H_{v}$-module over the $H_{v}$-ring $H$ if there is a map $\cdot H \times M \rightarrow M$ such that

$$
\begin{aligned}
& \forall a, b \in H \forall m, n \in M[a(m+n) \cap(a m+a n) \neq \emptyset, \\
&(a+b) m \cap(a m+b m) \neq \emptyset,(a b) m \cap a(b m) \neq \emptyset] .
\end{aligned}
$$

Right $H_{v}$-modules are defined analogously.

(2) Let $H$ be a Krasner hyperring. A canonical hypergroup $(M,+)$ is called a left $H$ hypermodule over the Krasner hyperring $H$ if there is a map $\cdot H \times M \rightarrow M$ such that

$$
\begin{aligned}
& \forall a, b \in H \forall m, n \in M[a(m+n)=a m+a n, \\
& (a+b) m=a m+b m,(a b) m=a(b m), 0 m=0] .
\end{aligned}
$$

Right $H$-hypermodules are defined analogously.

REMARK 2.7. $H_{v}$-modules were introduced together with $H_{v}$-groups and $H_{v}$-rings by Vougiouklis [38. The notion of hypermodules goes back to the early 1970s and the works of Mittas [24] and Stratigopoulos [36]. Just like in the case with ordinary modules, canonical hypergroups are $\mathbb{Z}$-hypermodules, Krasner hyperrings are hypermodules over themselves etc.

Definition 2.8. Let $H$ be an $H_{v}$-group. An $H_{v}$-subgroup is a subset $K \subseteq H$ which is an $H_{v}$-group itself.

Let $H$ be a canonical hypergroup. A canonical subhypergroup is a subset $K \subseteq H$ which is a canonical hypergroup itself.

Let $H$ be an $H_{v}$-ring and $M$ a left $H_{v}$-module over $H$. An $H_{v}$-submodule is a subset $N \subseteq M$ which is a left $H_{v}$-module over $H$ itself.

Let $H$ be a Krasner hyperring and $M$ a left $H$-hypermodule. An $H$-subhypermodule is a subset $N \subseteq M$ which is a left $H$-hypermodule itself. 
Lemma 2.9. Let $H$ be a Krasner hyperring, $M$ a left $H$-hypermodule, and let $A \subseteq M$. The smallest $H$-subhypermodule of $M$ containing the set $A$ is of the form $\left\{r_{1} a_{1}+\ldots+r_{n} a_{n}+a_{1}^{\prime}+\ldots+a_{n^{\prime}}^{\prime} \mid n, n^{\prime} \in \mathbb{N}, r_{1}, \ldots, r_{n} \in H, a_{1}, \ldots, a_{n}, a_{1}^{\prime}, \ldots, a_{n^{\prime}}^{\prime} \in A\right\}$. Proof. Firstly, observe that, as the intersection of any family of $H$-subhypermodules is an $H$-subhypermodule, it certainly makes sense to talk about smallest $H$-subhypermodules in the above sense. Now the argument is quite standard: let

$$
\begin{aligned}
\langle A\rangle=\left\{r_{1} a_{1}+\ldots+r_{n} a_{n}+a_{1}^{\prime}+\ldots+a_{n^{\prime}}^{\prime} \mid\right. & \\
n, n^{\prime} & \left.\in \mathbb{N}, r_{1}, \ldots, r_{n} \in H, a_{1}, \ldots, a_{n}, a_{1}^{\prime}, \ldots, a_{n^{\prime}}^{\prime} \in A\right\},
\end{aligned}
$$

and denote by $N$ the smallest $H$-subhypermodule containing the set $A$. That $\langle A\rangle$ is an $H$-subhypermodule is apparent, it is also clear that $A \subseteq\langle A\rangle$, and thus $N \subseteq\langle A\rangle$. Fix $m \in\langle A\rangle$. Then

$$
m \in r_{1} a_{1}+\ldots+r_{n} a_{n}+a_{1}^{\prime}+\ldots+a_{n^{\prime}}^{\prime}
$$

for some $n, n^{\prime} \in \mathbb{N}, r_{1}, \ldots, r_{n} \in H, a_{1}, \ldots, a_{n}, a_{1}^{\prime}, \ldots, a_{n^{\prime}}^{\prime} \in A$. But then $a_{1}, \ldots, a_{n}$, $a_{1}^{\prime}, \ldots, a_{n^{\prime}}^{\prime} \in A \subseteq N$ which is an $H$-subhypermodule, so that $r_{1} a_{1}+\ldots+r_{n} a_{n}+a_{1}^{\prime}+$ $\ldots+a_{n^{\prime}}^{\prime} \subseteq N$ and, consequently, $m \in N$.

Proposition 2.10. Let $H$ be a canonical hypergroup, let $K \subseteq H$ be a canonical subhypergroup. For an element $h \in H$ denote by $\bar{h}$ the coset $h+K$, consider the set

$$
H / K=\{\bar{h} \mid h \in H\},
$$

and for $\overline{h_{1}}, \overline{h_{2}} \in H / K$ define the hyperoperation $\oplus$ on $H / K$ by

$$
\overline{h_{1}} \oplus \overline{h_{2}}=\bigcup\left\{\bar{h} \mid h \in h_{1}+h_{2}\right\},
$$

as well as the element $\ominus \bar{h} \in H / K$ to be the coset of $-h, h \in H$. Then $H / K$ with $\oplus i s$ a canonical hypergroup.

See, for example, [3, Proposition 3.2] for a proof (as well as for other concepts of quotients of canonical hypergroups), although the result itself has been well-known for a much longer time.

Definition 2.11. Let $H$ be a canonical hypergroup, let $K \subseteq H$ be a canonical subhypergroup. The canonical hypergroup $H / K$ is called a quotient hypergroup.

Proposition 2.12. Let $H$ be a Krasner hyperring, $M$ a left $H$-hypermodule and $N \subseteq M$ an $H$-subhypermodule of $M$. Consider the canonical hypergroup $M / N$ and for $a \in H$, $\bar{m} \in M / N$ define the external operation $\cdot$ on $M / N$ by

$$
a \cdot \bar{m}=\overline{a \cdot m} \text {. }
$$

Then $M / N$ with $\cdot$ is a left $H$-hypermodule.

See [33, Proposition 3] for a proof.

Definition 2.13. Let $H$ be a Krasner hyperring, $M$ a left $H$-hypermodule and $N \subseteq M$ an $H$-subhypermodule of $M$. The hypermodule $M / N$ of Proposition 2.12 is called a quotient hypermodule. 


\section{DEFINITION 2.14 .}

I. Let $H_{1}, H_{2}$ be two $H_{v}$-groups or canonical hypergroups. A map $f: H_{1} \rightarrow H_{2}$ (which, if $H_{1}, H_{2}$ are canonical hypergroups, also satisfies $\left.f(0)=0\right)$ is called:

1. a weak homomorphism, abbreviated w-homomorphism, if

$$
\forall a, b \in H_{1}[f(a+b) \cap(f(a)+f(b)) \neq \emptyset],
$$

2. an inclusion homomorphism, abbreviated i-homomorphism, if

$$
\forall a, b \in H_{1}[f(a+b) \subseteq f(a)+f(b)]
$$

3. a strong homomorphism, abbreviated s-homomorphism, if

$$
\forall a, b \in H_{1}[f(a+b)=f(a)+f(b)]
$$

II. Let $H_{1}, H_{2}$ be two $H_{v}$-groups or canonical hypergroups. A map $f: H_{1} \rightarrow \mathcal{P}^{*}\left(H_{2}\right)$ (which, if $H_{1}, H_{2}$ are canonical hypergroups, also satisfies $f(0)=\{0\}$ ) is called:

1. a multivalued weak homomorphism, abbreviated mw-homomorphism, if

$$
\forall a, b \in H_{1}[f(a+b) \cap(f(a)+f(b)) \neq \emptyset],
$$

2. a multivalued inclusion homomorphism, abbreviated mi-homomorphism, if

$$
\forall a, b \in H_{1}[f(a+b) \subseteq f(a)+f(b)],
$$

3. a multivalued strong homomorphism, abbreviated ms-homomorphism, if

$$
\forall a, b \in H_{1}[f(a+b)=f(a)+f(b)] .
$$

Lemma 2.15. Let $H_{1}, H_{2}$ be canonical hypergroups, let $f: H_{1} \rightarrow H_{2}$ be $i$ - or s-homomorphism. Then

$$
\forall a \in H_{1}[f(-a)=-f(a)] .
$$

Proof. For a fixed $a \in H_{1}$, as $0 \in a-a$, one has

$$
f(0) \in f(a-a) \subseteq f(a)+f(-a)
$$

so that

$$
f(-a) \in f(0)-f(a)=0-f(a)=-f(a) .
$$

DEFINITION 2.16.

(1) Let $H$ be an $H_{v}$-ring, let $M_{1}, M_{2}$ be two left $H_{v}$-modules. A $w$-, $i$-, $s$-, $m w-, m i$ - or ms-homomorphism $f: M_{1} \rightarrow M_{2}$ is a w-, i-, s-, mw-, mi- or ms-homomorphism of underlying $H_{v}$-groups such that

$$
\forall a \in H \forall m \in M_{1}[a f(m)=f(a m)] .
$$

(2) Let $H$ be a Krasner hyperring, let $M_{1}, M_{2}$ be two left $H$-hypermodules. A $w$-, $i$-, $s-, m w-, m i-$ or ms-homomorphism $f: M_{1} \rightarrow M_{2}$ is a w-, i-, s-, mw-, mi- or mshomomorphism of underlying canonical hypergroups such that

$$
\forall a \in H \forall m \in M_{1}[a f(m)=f(a m)] .
$$




\section{NOTATION 2.17 .}

I. Let $H$ be an $H_{v}$-ring.

1. The category of left $H_{v}$-modules over $H$ with mw-homomorphisms and composition of morphisms $M \stackrel{f}{\rightarrow} N$ and $N \stackrel{g}{\rightarrow} K$ defined by

$$
g f(m)=\bigcup_{n \in f(m)} g(n)
$$

will be denoted by $H-H_{v}^{w}$ Mod.

2. The category of left $H_{v}$-modules over $H$ with mi-homomorphisms will be denoted by $H-H_{v}^{i} \mathcal{M o d}$.

3. The category of left $H_{v}$-modules over $H$ with ms-homomorphisms will be denoted by $H-H_{v}^{s}$ Mod.

4. The category of left $H_{v}$-modules over $H$ with w-homomorphisms will be denoted by $H-h_{v}^{w}$ Mod.

5. The category of left $H_{v}$-modules over $H$ with i-homomorphisms will be denoted by $H-h_{v}^{i}$ Mod.

6. The category of left $H_{v}$-modules over $H$ with s-homomorphisms will be denoted by $H-h_{v}^{s}$ Mod.

II. Let $H$ be a Krasner hyperring.

1. The category of left hypermodules over $H$ with mw-homomorphism will be denoted by $H-H^{w}$ Mod.

2. The category of left hypermodules over $H$ with mi-homomorphism will be denoted by $H-H^{i} \mathcal{M}$ od.

3. The category of left hypermodules over $H$ with ms-homomorphism will be denoted by $H-H^{s}$ Mod.

4. The category of left hypermodules over $H$ with w-homomorphism will be denoted by $H-h^{w}$ Mod.

5. The category of left hypermodules over $H$ with i-homomorphism will be denoted by $H-h^{i}$ Mod.

6. The category of left hypermodules over $H$ with s-homomorphism will be denoted by $H-h^{s}$ Mod.

For categories $\mathcal{C}$ and $\mathcal{D}$ we shall write $\mathcal{C} \prec \mathcal{D}$ to denote that $\mathcal{C}$ is a subcategory of $\mathcal{D}$. The following observation follows straight from the above definitions:

Proposition 2.18. If $H$ is an $H_{v}$-ring then

$$
H-H_{v}^{s} \mathcal{M o d} \prec H-H_{v}^{i} \mathcal{M o d} \prec H-H_{v}^{w} \mathcal{M o d}
$$

and

$$
H-h_{v}^{s} \mathcal{M o d} \prec H-h_{v}^{i} \mathcal{M o d} \prec H-h_{v}^{w} \mathcal{M o d} .
$$

If $H$ is a Krasner hyperring then

$$
H-H^{s} \mathcal{M o d} \prec H-H^{i} \mathcal{M} \text { od } \prec H-H^{w} \mathcal{M o d}
$$

and

$$
H-h^{s} \mathcal{M o d} \prec H-h^{i} \mathcal{M o d} \prec H-h^{w} \mathcal{M} \text { od }
$$




\section{Monics, epics, isos and factorizations}

Proposition 3.1. Let $H$ be a Krasner hyperring, let $M, N$ be $H$-hypermodules.

1. For a morphism $f: M \rightarrow N$ in $H-h^{i} \mathcal{M}$ od the following are equivalent:

i. $f$ is a monic;

ii. $f$ is injective.

2. For a morphism $f: M \rightarrow N$ in $H-h^{s} \mathcal{M o d}$ the following are equivalent:

i. $f$ is a monic;

ii. $f$ is injective;

iii. $\operatorname{Ker}(f)=\{m \in M \mid f(m)=0\}=\{0\}$.

See [33, Proposition 4] and [18, Theorem 3.3] for a proof.

Proposition 3.2. Let $H$ be a Krasner hyperring, let $M, N$ be $H$-hypermodules.

1. For a morphism $f: M \rightarrow N$ in $H-h^{i} \mathcal{M o d}$, if $f$ is surjective, then it is an epic.

2. For a morphism $f: M \rightarrow N$ in $H-h^{s} \mathcal{M}$ od the following are equivalent:

i. $f$ is an epic;

ii. $f$ is surjective.

See [33, Proposition 5] and [18, Theorem 3.4] for a proof. As functions between sets are injective if and only if they have left inverses, and are surjective if and only if they have right inverses, the following is now clear:

Corollary 3.3. Let $H$ be a Krasner hyperring. The category $H-h^{s} \mathcal{M o d}$ is balanced, i.e. isos are bijections.

Proposition 3.4. Let $H$ be a Krasner hyperring, let $M, N$ be H-hypermodules.

1. For a morphism $f: M \rightarrow N$ in $H-h^{i} \mathcal{M o d}$ and a $H$-subhypermodule $K \subseteq \operatorname{Ker}(f)$, there exists exactly one morphism $g: M / K \rightarrow N$ such that $f=g \circ \kappa$, where $\kappa: M \rightarrow M / K$ is the canonical $i$-homomorphism given by $\kappa(m)=m+K$. In other words, the following diagram is commutative:

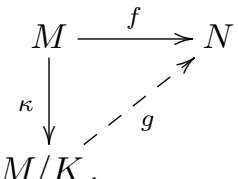

$$
M / K \text {. }
$$

2. For a morphism $f: M \rightarrow N$ in $H-h^{s} \mathcal{M o d}$ and a $H$-subhypermodule $K \subseteq \operatorname{Ker}(f)$, there exists exactly one morphism $g: M / K \rightarrow N$ such that $f=g \circ \kappa$, where $\kappa: M \rightarrow$ $M / K$ is the canonical s-homomorphism given by $\kappa(m)=m+K$. In other words, the following diagram is commutative:

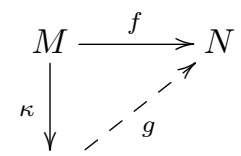

$M / K$. 
Moreover, if $f$ is an epic, then $g$ is an epic, and if $K=\operatorname{Ker}(f)$, then $g$ is a monic.

Consequently, if $f$ is an epic and $K=\operatorname{Ker}(f)$, then $g$ is an iso.

See [33, Theorem 1] and [18, Corollary 3.2] for a proof. In particular in $H-h^{s} \mathcal{M o d}$ every morphism $f$ factors through $\operatorname{Ker}(f)$.

\section{Products, coproducts and free objects}

Proposition 4.1. Let $H$ be a Krasner hyperring. Products exist in the categories $H-h^{s} \mathcal{M o d}$ and $H-H^{s} \mathcal{M o d}$ and coproducts exist in the category $H-H^{s} \mathcal{M o d}$.

See [1, Theorem 3.14 and Theorem 3.17] for a proof in the $H-H^{s}$ Mod case that easily translates to the $H-h^{s}$ Mod one for products. These are standard constructions: for a Krasner hyperring $H$ and a family of $H$-hypermodules $\left\{M_{i} \mid i \in I\right\}$ consider the Cartesian product $\prod_{i \in I} M_{i}$ of sets and define

$$
\left(m_{i}\right)_{i \in I}+\left(m_{i}^{\prime}\right)_{i \in I}=\left\{\left(n_{i}\right)_{i \in I} \mid n_{i} \in m_{i}+m_{i}^{\prime}, i \in I\right\} \text { for }\left(m_{i}\right)_{i \in I}, \quad\left(m_{i}^{\prime}\right)_{i \in I} \in \prod_{i \in I} M_{i},
$$

as well as

$$
a\left(m_{i}\right)_{i \in I}=\left(a m_{i}\right)_{i \in I} \text { for } a \in H,\left(m_{i}\right)_{i \in I} \in \prod_{i \in I} M_{i} .
$$

Then $\prod_{i \in I} M_{i}$ is an $H$-hypermodule which together with canonical projections $\pi_{k}: \prod_{i \in I} M_{i} \rightarrow M_{k}$ given by

$$
\pi_{k}\left(\left(m_{i}\right)_{i \in I}\right)=m_{k},
$$

for $k \in I$, forms a product in the categories $H-h^{s} \mathcal{M o d}$ and $H-H^{s} \mathcal{M o d}$ : if $M$ is another $H$-hypermodule with a family of ms- or s-homomorphisms $\left\{f_{k}: M \rightarrow M_{k} \mid k \in I\right\}$, then the unique ms- or s-homomorphism $f: M \rightarrow \prod_{i \in I} M_{i}$ such that $\pi_{k} \circ f=f_{k}, k \in I$, is given by

$$
f(m)=\left(f_{i}(m)\right)_{i \in I} \text { for } m \in M
$$

Similarly, the set

$$
\sum_{i \in I} M_{i}=\left\{\left(m_{i}\right)_{i \in I} \in \prod_{i \in I} M_{i} \mid m_{i} \neq 0 \text { for only finitely many } i \in I\right\}
$$

is an $H$-hypermodule with the above defined operations that along with canonical injections $\iota_{k}: M_{k} \rightarrow \sum_{i \in I} M_{i}$ given by

$$
\iota_{k}(m)=\left(\bar{m}_{i}\right)_{i \in I}
$$

where

$$
\bar{m}_{i}= \begin{cases}m, & \text { if } i=k, \\ 0, & \text { if } i \neq k,\end{cases}
$$

for $k \in I$, constitutes a coproduct in the category $H-H^{s} \mathcal{M o d}$ : if $M$ is an $H$-hypermodule with a family of ms-homomorphisms $\left\{g_{k}: M_{k} \rightarrow M \mid k \in I\right\}$, then the uniquely defined ms-homomorphism $g: \sum_{i \in I} M_{i} \rightarrow M$ such that $g \circ \iota_{k}=g_{k}, k \in I$, is given by the formula

$$
g\left(\left(m_{i}\right)_{i \in I}\right)=g_{i_{1}}\left(m_{i_{1}}\right)+\ldots+g_{i_{l}}\left(m_{i_{l}}\right),
$$


where $\left\{i_{1}, \ldots, i_{l}\right\}=\left\{i \in I \mid m_{i} \neq 0\right\}$ (note that there is no reason why this map would be an s-homomorphism).

Proposition 4.2. Let $H$ be a Krasner hyperring. Free objects do not exist in the category $H-h^{i}$ Mod.

See [25. Theorem 2.1] for a proof of the corresponding result for hypergroups, that readily applies to hypermodules. Various notions of partial freeness in the categories $H-H^{i} \mathcal{M o d}$ and $H-H^{s} \mathcal{M}$ od are also discussed by Ameri and Shojaei in [2]. "Free hypermodules", although not free objects in the category theoretical meaning, were initially considered by Massouros in [21].

\section{Subobjects and quotient objects}

Proposition 5.1. Let $H$ be a Krasner hyperring. In the category $H-h^{s}$ Mod subobjects are $H$-subhypermodules together with the inclusion maps.

Proposition 5.2. Let $H$ be a Krasner hyperring. In the category $H-h^{s} \mathcal{M o d}$ quotient objects are quotient hypermodules together with the canonical projection maps.

See [18, Theorem 4.1] for a proof of both results.

\section{Kernels, coimages, images and cokernels}

Proposition 6.1. Let $H$ be a Krasner hyperring. The categories $H-H^{s} \mathcal{M o d}$, $H-H^{i} \mathcal{M o d}, H-H^{w} \mathcal{M o d}, H-h^{s} \mathcal{M o d}, H-h^{i} \mathcal{M o d}$ and $H-h^{w} \mathcal{M o d}$ all have the zero object $0:=\{0\}$.

Proof. It suffices to check that if $f: M \rightarrow N$ is a ms-, mi-, mw-, s-, i- or w-homomorphism of $H$-hypermodules, then $f(0)=0$, which is the case as:

$$
f(0)=f(0 m)=0 f(m)=0,
$$

for any choice of $m \in M$.

Proposition 6.2.

1. Let $H$ be an $H_{v}$-ring. Equalizers exist in the categories $H-H_{v}^{s} \mathcal{M o d}$ and $H-h_{v}^{s} \mathcal{M o d}$.

2. Let $H$ be a Krasner hyperring. Equalizers exist in the categories $H-H^{s} \mathcal{M o d}$ and $H-h^{s}$ Mod.

Proof. The arguments are basically identical for both $H_{v}$-modules and $H$-hypermodules, we shall carry them for $H$-hypermodules here. Let $H$ be a Krasner hyperring, let $M, N$ be left $H$-hypermodules, let $f, g: M \rightarrow N$ be s-homomorphisms. The set

$$
\operatorname{Eq}(f, g)=\{m \in M \mid f(m)=g(m)\}
$$

is an $H$-subhypermodule of $M$. Indeed, if $m_{1}, m_{2} \in \mathrm{Eq}(f, g)$, then

$$
f\left(m_{1}+m_{2}\right)=f\left(m_{1}\right)+f\left(m_{2}\right)=g\left(m_{1}\right)+g\left(m_{2}\right)=g\left(m_{1}+m_{2}\right),
$$

so that $m_{1}+m_{2} \in \operatorname{Eq}(f, g)$, and if $m \in M$ and $a \in H$, then

$$
f(a m)=a f(m)=a g(m)=g(a m),
$$


so that $a m \in \operatorname{Eq}(f, g)$. The inclusion map eq $(f, g): \operatorname{Eq}(f, g) \hookrightarrow M$ is clearly an s-homomorphism and $f \circ \mathrm{eq}(f, g)=g \circ \mathrm{eq}(f, g)$. If $E$ is another $H$-hypermodule and $e: E \rightarrow M$ another s-homomorphism such that $f \circ e=g \circ e$, then for the corestriction $e l_{\mathrm{Eq}(f, g)}$ clearly one has eq $(f, g) \circ e \mathrm{~L}_{\mathrm{Eq}(f, g)}=e$. Moreover, if $\bar{e}: E \rightarrow \operatorname{Eq}(f, g)$ is another s-homomorphism such that $\mathrm{eq}(f, g) \circ \bar{e}=e$ then, since eq $(f, g)$ is a monic as an injective function between sets, $\bar{e}=e l_{\operatorname{Eq}(f, g)}$.

ExAMPLE 6.3. Let $H$ be a Krasner hyperring. Equalizers do not exist in the categories $H-H^{i} \mathcal{M}$ od and $H-h^{i} \mathcal{M}$ od. Indeed, we shall see this for the category $H-h^{i} \mathcal{M}$ od. Consider the three-element "sign hypergroup" $Q_{2}$ consisting of the elements $-1,0,1$ and with addition defined by $0+m=m$, for all $m \in Q_{2}, m+m=m$, for all $m \in Q_{2}$, and

$$
1+(-1)=(-1)+1=\{-1,0,1\} .
$$

One easily checks that this is, indeed, a hypergroup (see, for example, [19] or [37, Section 3.5]). Next, let $M$ be the three-element hypergroup $M=\{0,1,2\}$ with + defined by $0+m=m$, for all $m \in M$, and

$$
1+1=2, \quad 1+2=2+1=\{0,1\}, \quad 2+2=\{1,2\}
$$

(see [37, Section 3.5]), and let $\mathbb{R}_{\geqslant 0}=\{m \in \mathbb{R} \mid m \geqslant 0\}$ be the "ultratriangle hypergroup" (see [37, Section 5.2]) with addition defined as follows:

$$
m+n= \begin{cases}\max (m, n), & \text { if } m \neq n, \\ {[0, m],} & \text { if } m=n .\end{cases}
$$

Consider two maps $f, g: \mathbb{R}_{\geqslant 0} \rightarrow \mathbb{R}_{\geqslant 0}, f$ being just the identity, and $g$ the square function:

$$
g(m)=m^{2} \text { for all } m \in \mathbb{R}_{\geqslant 0} .
$$

They are both easily checked to be i-homomorphisms. Furthermore, consider the map $e_{1}: Q_{2} \rightarrow \mathbb{R}_{\geqslant 0}$ given by

$$
e_{1}(0)=0, \quad e_{1}(-1)=e_{1}(1)=1,
$$

and the map $e_{2}: M \rightarrow \mathbb{R}_{\geqslant 0}$ given by

$$
e_{2}(0)=0, \quad e_{2}(1)=e_{2}(2)=1 .
$$

A straightforward verification shows that they are both i-homomorphisms and that

$$
f \circ e_{1}=g \circ e_{1} \quad \text { and } \quad f \circ e_{2}=g \circ e_{2} .
$$

However, as one can easily check, there are no i-homomorphisms $Q_{2} \rightarrow M$ other than the zero map.

Proposition 6.4. Let $H$ be a Krasner hyperring. Kernels exist in the categories $H-H^{s} \mathcal{M o d}, H-H^{i} \mathcal{M o d}, H-h^{s} \mathcal{M o d}$ and $H-h^{i} \mathcal{M o d}$.

Proof. For $H$-hypermodules $M, N$ and for a ms-, mi-, s- or i-homomorphism $f: M \rightarrow N$

$$
\operatorname{Ker}(f)=\{m \in M \mid f(m)=0\}
$$

with the inclusion map $\operatorname{ker}(f): \operatorname{Ker}(f) \hookrightarrow M$ is the equalizer of the pair $f$ and $0_{M, N}$ : $M \rightarrow N$-for a ms- or s-homomorphism $f$ this is clear by Proposition 6.2, and for a mi- 
or i-homomorphism $f$, it suffices to check that $\operatorname{Ker}(f)$ is an $H$-subhypermodule, which is apparent: if $m_{1}, m_{2} \in \operatorname{Ker}(f)$, then

$$
f\left(m_{1}+m_{2}\right) \subseteq f\left(m_{1}\right)+f\left(m_{2}\right)=0+0=0,
$$

so that $m_{1}+m_{2} \in \operatorname{Ker}(f)$, and if $a \in H, m \in \operatorname{Ker}(f)$, then

$$
f(a m)=a f(m)=a 0=0,
$$

so that $a m \in \operatorname{Ker}(f)$.

Proposition 6.5. Let $H$ be a Krasner hyperring. Coimages exist in the category $H-h^{s}$ Mod.

See [18, Theorem 4.2.(2)] for a proof using the standard argument relying on the factorization theorem of Proposition 3.4 The coimage of an s-homomorphism $f: M \rightarrow N$, where $M, N$ are left $H$-hypermodules, is

$$
\operatorname{Coim}(f)=M / \operatorname{Ker}(f)
$$

and $\operatorname{coim}(f): M \rightarrow \operatorname{Coim}(f)$ is the canonical s-homomorphism given by $\operatorname{coim}(f)(m)=$ $m+\operatorname{Ker}(f)$.

Proposition 6.6. Let $H$ be a Krasner hyperring. Images exist in the categories $H-H^{s} \mathcal{M o d}$ and $H-h^{s} \mathcal{M o d}$.

See [18, Theorem 4.2.(1)] for a proof in the $H-h^{s} \mathcal{M o d}$ case that carries automatically to the $H-H^{s}$ Mod case. The image of an s-homomorphism (ms-homomorphism) $f: M \rightarrow N$, where $M, N$ are left $H$-hypermodules, is just

$$
\operatorname{Im}(f)=\{f(m) \mid m \in M\} \quad\left(\operatorname{Im}(f)=\bigcup_{m \in M}\{f(m) \mid m \in M\}\right),
$$

and $\operatorname{im}(f): \operatorname{Im}(f) \hookrightarrow N$ is the inclusion map.

ExAmPLE 6.7. Let $H$ be a Krasner hyperring. Coequalizers do not exist in the categories $H-H^{s} \mathcal{M o d}, H-H^{i} \mathcal{M o d}, H-h^{s} \mathcal{M o d}$ and $H-h^{i} \mathcal{M}$ od. We shall carry the argument for the category $H-h^{s} \mathcal{M}$ od. Denote by $\mathcal{R}$ the set of non-zero reals $\mathbb{R}^{\times}$together with multivalued addition defined as follows:

$$
m+n= \begin{cases}\{m n,-m n\}, & \text { if } m \neq 1 \wedge n \neq 1, \\ m n, & \text { if } m=1 \vee n=1 .\end{cases}
$$

By means of tedious, but straightforward computation (which, in particular, involves going through eight different cases to verify the law of associativity) one checks that $\mathcal{R}$ is a canonical hypergroup, hence a $\mathbb{Z}$-hypermodule. Consider the map $\mu: \mathcal{R} \rightarrow \mathcal{R}$ given by

$$
\mu(m)=\left\{\begin{array}{ll}
-m, & \text { if } m \neq \pm 1, \\
m, & \text { if } m= \pm 1,
\end{array} \quad \text { for } m \in \mathcal{R} .\right.
$$

Again, a routine verification shows that $\mu$ is an s-homomorphism. Now take two maps $f, g: \mathcal{R} \times \mathcal{R} \rightarrow \mathcal{R} \times \mathcal{R}$ defined by

$$
f(m, n)=(m, n),
$$


and

$$
g(m, n)=(\mu(m), n),
$$

respectively, for $(m, n) \in \mathcal{R} \times \mathcal{R}$. They are both easily seen to be s-homomorphism, and they are clearly coequalized on the second coordinate by the identity s-homomorphism $c_{1}: \mathcal{R} \times \mathcal{R} \rightarrow \mathcal{R}, c_{1}(m, n)=n:$

$$
c_{1} \circ f=c_{1} \circ g .
$$

Denote by $\mathbb{R}_{+}$the multiplicative group (hence a hypergroup, hence a $\mathbb{Z}$-hypermodule) of positive reals and consider the absolute value on the first coordinate map $c_{2}: \mathcal{R} \times \mathcal{R} \rightarrow \mathbb{R}_{+}$ given by

$$
c_{2}(m, n)=|m| \text { for }(m, n) \in \mathcal{R} \times \mathcal{R} .
$$

It is easily checked to be an s-homomorphism such that

$$
c_{2} \circ f=c_{2} \circ g .
$$

However, there is no s-homomorphism $\bar{c}: \mathbb{R}_{+} \rightarrow \mathcal{R}$ such that $\bar{c} \circ c_{2}=c_{1}$, for if such a $\bar{c}$ existed, then

$$
\bar{c}(1)=\bar{c} \circ c_{2}(-1,-1)=c_{1}(-1,-1)=-1,
$$

which is impossible, as s-homomorphisms carry identities to identities.

Proposition 6.8. Let $H$ be a Krasner hyperring. Cokernels exist in the categories $H-H^{s} \mathcal{M o d}$ and $H-h^{s} \mathcal{M o d}$.

See [1, Theorem 3.18] for a proof in the $H-H^{s} \mathcal{M o d}$ case and [18, Theorem 4.3] in the $H-h^{s}$ Mod case. The cokernel of an ms- or s-homomorphism $f: M \rightarrow N$ is just

$$
\text { Coker }(f)=N / \operatorname{Im}(f),
$$

and coker $(f): N \rightarrow \operatorname{Coker}(f)$ is the canonical projection.

Corollary 6.9. Let $H$ be a Krasner hyperring. Pullbacks exist in the categories $H-H^{s}$ Mod and $H-h^{s} \mathcal{M o d}$. Pushouts do not exist in the categories $H-H^{s} \mathcal{M o d}$, $H-H^{i} \mathcal{M o d}, H-h^{s} \mathcal{M o d}, H-h^{i} \mathcal{M o d}$.

Proposition 6.10. Let $H$ be a Krasner hyperring. The categories $H-H^{s} \mathcal{M o d}$ and $H-h^{s}$ Mod are normal and conormal, i.e. monics are kernels and epics are cokernels.

See [18, Theorem 4.3] for a proof in the $H-h^{s} \mathcal{M}$ od case that also applies to $H-H^{s} \mathcal{M}$ od.

7. Exactness and final remarks. In view of the concluding results of the previous section we have, in particular, the following:

Corollary 7.1. Let $H$ be a Krasner hyperring. The categories $H-H^{s} \mathcal{M o d}$ and $H-h^{s} \mathcal{M o d}$ are Puppe exact, i.e. have zero object, kernels and cokernels, are normal and conormal, and every morphism has an epi-mono factorization.

Recall that Puppe exact categories were introduced in the 1960s 30, and, roughly speaking, are Abelian categories without additivity. The categories of hypermodules discussed in this survey are clearly not Abelian, as binary products are not coproducts, so that it seems that Puppe exactness is the best attainable notion of exactness here. Nevertheless, a version of homological algebra for Puppe exact categories can be developed, 
as shown by Mitchell in his classical textbook 22. Also see a relatively recent preprint by Grandis [13] for generalizations of Puppe exactness and related concepts (as well as references to his earlier work).

Various weak notions of additivity in categories of hypermodules have been studied by numerous authors, see, for example, 32. It is not clear if these notions would lead to the development of a well-behaved homological algebra. Also, see [26] for a very well-known survey on hyperrings by Nakassis, as well as [28 for a more general approach to the categorical aspects of hyperstructures through multialgebras, and [27] for most recent results on Krasner hyperrings.

\section{References}

[1] R. Ameri, On categories of hypergroups and hypermodules, J. Discrete Math. Sci. Cryptogr. 6 (2003), 121-132.

[2] R. Ameri, H. Shojaei, Various kinds of freeness in the categories of Krasner hypermodules, Intl. J. Anal. Appl. 16 (2018), 793-808.

[3] R. Ameri, H. Shojaei, Various kinds of quotient of a canonical hypergroup, Sigma J. Eng. Nat. Sci. 9 (2018), 133-141.

[4] S. D. Comer, Extension of polygroups by polygroups and their representations using color schemes, in: Universal Algebra and Lattice Theory (Puebla, 1982), Lecture Notes in Math. 1004, Springer, Berlin, 1983, 91-103.

[5] P. Corsini, Hypergroupes et groupes ordonnés, Rend. Sem. Mat. Univ. Padova 48 (1972), 189-204.

[6] P. Corsini, A contribution to hypergroup theory, Atti Soc. Peloritana Sci. Fis. Mat. Natur. 26 (1980), 347-362.

[7] P. Corsini, Prolegomena of Hypergroup Theory, Supplement to Riv. Mat. Pura Appl., Aviani Editore, Tricesimo, 1993.

[8] P. Corsini, V. Leoreanu, Applications of Hyperstructure Theory, Adv. Math. (Dordr.) 5, Kluwer Acad. Publ., Dordrecht, 2003.

[9] B. Davvaz, Polygroup Theory and Related Systems, World Scientific, Hackensack, NJ, 2013.

[10] B. Davvaz, I. Cristea, Fuzzy Algebraic Hyperstructures. An Introduction, Stud. Fuzziness Soft Comput. 321, Springer, Cham, 2015.

[11] B. Davvaz, V. Leoreanu-Fotea, Hyperring Theory and Applications, International Academic Press, Palm Harbor, FL, 2007.

[12] M. De Salvo, Hyperrings and Hyperfields, Ann. Sci. Univ. Clermont-Ferrand II Math. 22 (1984), 89-107.

[13] M. Grandis, Homotopy spectral sequences, arXiv:1007.0632

[14] L. Konguetsof, Th. Vougiouklis, Constructions d'hyperanneaux a partir d'anneaux, Acta Univ. Carolin. Math. Phys. 28 (1987), 9-13.

[15] M. Koskas, Groupoides, demi-hypergroupes et hypergroupes, J. Math. Pures Appl. (9) 49 (1970), 155-192.

[16] M. Krasner, Approximation des corps valués complets de caractéristique $p \neq 0$ par ceux de caractéristique 0, in: Colloque d'algebre supérieure, tenu a Bruxelles du 19 au 22 décembre 1956, Centre Belge de Recherches Mathématiques, Établissements Ceuterick, Louvain; Librairie Gauthier-Villars, Paris, 1957, 129-206. 
[17] V. Leoreanu, Une note sur les demi-hypergroupes qui sont quasi-hypergroupes d'un côté, in: Algebraic Hyperstructures and Applications (Prague, 1996), Democritus Univ. Thrace, Alexandroupolis, 1997, 85-87.

[18] A. Madanshekaf, Exact category of hypermodules, Int. J. Math. Math. Sci. 2006, art. 31368.

[19] M. Marshall, Real reduced multirings and multifields, J. Pure Appl. Algebra 205 (2006), $452-468$.

[20] F. Marty, Sur une généralisation de la notion de groupe, in: Congrés des Mathématiciens Scandinaves Tenu a Stockholm, Stockholm, 1934, 45-49.

[21] Ch. Massouros, Free and cyclic hypermodules, Ann. Mat. Pura Appl. (4) 150 (1988), 153166.

[22] B. Mitchell, Theory of Categories, Pure and Applied Math. XVII, Academic Press, New York, 1965.

[23] J. Mittas, Hyperanneaux et certaines de leurs propriétés, C. R. Acad. Sci. Paris Sér. A-B, 269 (1969), A623-A626.

[24] J. Mittas, Espaces vectorieles sur un hypercorps. Introduction des hyperspaces affines et euclidiens, Math. Balkanica 5 (1975), 199-211.

[25] S. Sh. Mousavi, M. Jafarpour, On free and weak free (semi)hypergroups, Algebra Colloq. 18 (2011), Special Issue 1, 873-880.

[26] A. Nakassis, Recent results in hyperring and hyperfield theory, Internat. J. Math. Math. Sci. 11 (1988), 209-220.

[27] M. Norouzi, I. Cristea, Transitivity of the $\varepsilon_{m}$-relation on (m-idempotent) hyperrings, Open Math. 16 (2018), 1012-1021.

[28] C. Pelea, Hyperrings and $\alpha^{*}$-relations. A general approach, J. Algebra 383 (2013), 104-128.

[29] R. Procesi, R. Rota, Le spectre premier d'un hyperanneau multiplicatif, in: Hypergroups, other multivalued structures and their applications (Udine, 1985) (Italian), Univ. Studi Udine, Udine, 1985, 121-130.

[30] D. Puppe, Korrespondenzen in abelschen Kategorien, Math. Ann. 148 (1962), 1-30.

[31] R. Rota, Multiplicative hyperrings, Rend. Mat. (7) 2 (1982), 711-724.

[32] H. Shojaei, R. Ameri, S. Hošková-Mayerová, Pre-semihyperadditive categories, An. Ştiinţ. Univ. "Ovidius" Constanţa Ser. Mat. 27 (2019), 269-288.

[33] H. Shojaei, D. Fasino, Isomorphism theorems in the primary categories of Krasner hypermodules, Symmetry 11 (2019), 687.

[34] S. Spartalis, Homomorphisms on $(H, R)-H_{V}$-rings, in: Algebraic Hyperstructures and Applications (Prague, 1996), Democritus Univ. Thrace, Alexandroupolis, 1997, 133-138.

[35] S. Spartalis, Th. Vougiouklis, The fundamental relations of $H_{v}$-rings, Riv. Mat. Pura Appl. 14 (1994), 7-20.

[36] D. Stratigopoulos, Hyperanneaux non commutatifs: Hyperanneaux, hypercorps, hypermodules, hyperespaces vectoriels et leurs propriétés élémentaires, C. R. Acad. Sci. Paris Sér. A-B, 269 (1969), A489-A492.

[37] O. Viro, Hyperfields for tropical geometry I. Hyperfields and dequantization, arXiv:1006.3034

[38] Th. Vougiouklis, The fundamental relation in hyperrings. The general hyperfield, in: Algebraic Hyperstructures and Applications (Xánthi, 1990), World Sci. Publ., Teaneck, NJ, 1991, 203-211. 